\title{
DISTRIBUIÇÃO GEOGRÁFICA DA FORMAÇÃO EM FISIOTERAPIA NO BRASIL: CRESCIMENTO DESORDENADO E DESIGUALDADE REGIONAL
}

\author{
GEOGRAPHIC DISTRIBUTION OF TRAINING IN PHYSICAL THERAPY IN \\ BRAZIL: DISORDERED GROWTH AND REGIONAL INEQUALITY
}

DISTRIBUCIÓN GEOGRÁFICA DE LA FORMACIÓN EN FISIOTERAPIA EN

BRASIL: CRECIMIENTO DESORDENADO Y DESIGUALDAD REGIONAL

\author{
Lydia Chrismann Espíndola Koetz ${ }^{1}$ \\ Eduardo Périco ${ }^{2}$ \\ Magali Quevedo Grave ${ }^{3}$
}

Resumo O crescimento vertiginoso no número de escolas de Fisioterapia no Brasil carece de análise. Entre 1995 e 2008 houve um aumento de $476 \%$ do número de cursos localizados em grandes cidades e em instituições de ensino privadas. Com o objetivo de analisar a distribuição de escolas de Fisioterapia por regiões do Brasil, identificando-se as instituições com ensino presencial e a distância, realizou-se um estudo quantitativo, exploratório, transversal, a partir de dados secundários do site e-MEC em 2015. Foram relacionados o número de vagas e a distribuição de escolas, conforme estimativa populacional. Os cursos de Fisioterapia estão concentrados em grandes cidades, dificultando o acesso da população do interior. A maior concentração ocorre nos estados de São Paulo (239 cursos), Minas Gerais (152 cursos) e Paraná (120 cursos), onde também há o maior número de profissionais. Na análise de distribuição de cursos, identificou-se a necessidade de investir na abertura de cursos na região Norte. O crescimento desenfreado de escolas de Fisioterapia, somado à falta de critérios para a abertura de novos cursos no país, reitera as desigualdades na distribuição de profissionais.

Palavras-chave fisioterapia; ensino a distância; ensino.
Abstract The incredible growth in the number of Physical Therapy schools in Brazil needs to be analyzed. There was a 476 percent increase in the number of courses located in major cities and in private educational institutions between 1995 and 2008. Aiming to analyze the distribution of Physical Therapy schools in the different Brazilian regions, identifying the institutions offering presence and distance education, a quantitative, exploratory, and cross-sectional study was carried out based on secondary data collected from the e-MEC site in 2015. The number of openings and school distribution were listed based on population estimates. Physiotherapy courses are concentrated in large cities, making it difficult for the population in the interior to have access to them. The highest concentration is in the states of São Paulo (239 courses), Minas Gerais (152 courses), and Paraná (120 courses), where most professionals are also located. Course distribution analysis identified the need to invest in opening courses in Northern Brazil. The unbridled growth of Physical Therapy schools, coupled with the lack of criteria for opening new courses in the country, reiterates inequalities in the distribution of professionals.

Keywords physiotherapy; distance learning; education. 


\section{Introdução}

A primeira proposta de currículo mínimo para formação em Fisioterapia foi publicada via portaria ministerial n. 511, em 1964, baseada no parecer n. 388/63, no qual constava a primeira definição do fisioterapeuta, como sendo um profissional auxiliar que desempenhava tarefas terapêuticas mediante responsabilidade de um médico. Os primeiros cursos de Fisioterapia tinham período previsto de três anos letivos. O currículo era composto por matérias básicas, como fundamentos da Fisioterapia, ética, administração, e específicas, como as disciplinas de Fisioterapia geral e aplicada. Conforme Oliveira (2002), ao analisar os currículos daquele período, era perceptível o quanto se pretendia valorizar a profissão médica, por este motivo os cursos tinham curta duração e alcunhavam-se como técnicos em Fisioterapia.

Em 1983, a publicação da resolução n. 4 do Conselho Federal de Educação estabeleceu um currículo mínimo para graduação em Fisioterapia. Com a participação de profissionais reconhecidos da área, foram definidos quatro ciclos para a formação em saúde: o primeiro, matérias biológicas e patologias; o segundo, com disciplinas de cunho geral como sociologia e antropologia, introdução à saúde, metodologia da pesquisa; o terceiro, composto de disciplinas pré-profissionalizantes como história e fundamento da Fisioterapia, avaliação funcional e bases e métodos de avaliação; e o quarto, com matérias profissionalizantes como neurologia, ortopedia e traumatologia, reumatologia, condições gineco-obstétricas e os estágios supervisionados (Haddad, 2006). A organização curricular demonstra o quanto o ensino em Fisioterapia neste período era voltado para a atenção curativa e tecnicista (Bispo Júnior, 2013).

Entre os anos de 1995 e 2008, a expansão do número de cursos de Fisioterapia foi expressiva, passando de 63 para 479, respectivamente, um incremento de $476 \%$. Essa expansão ocorreu em grandes centros urbanos e principalmente em instituições de ensino privadas (Bispo Júnior, 2009).

Em 1996, a partir da Lei de Diretrizes e Bases (LDB) n. 9394/96, é incorporada a noção de prevenção nas estruturas curriculares para o curso de Fisioterapia, estimulando a discussão entre os diferentes atores, tais como o Ministério da Educação e Cultura (MEC), Coffito (Conselho Federal de Fisioterapia e Terapia Ocupacional), Crefito (Conselho Regional de Fisioterapia e Terapia Ocupacional), Instituições de Ensino Superior (IES), para formulação das Diretrizes Curriculares Nacionais (DCN) para formação em Fisioterapia (Haddad et al., 2006).

Esta discussão resultou na publicação das diretrizes curriculares descritas pelo Conselho de Educação em 2002. A partir deste período, a formação em Fisioterapia visa contemplar as ações descritas pelo Sistema Único de Saúde (SUS). O perfil do fisioterapeuta é de um profissional generalista, humanístico, crítico e reflexivo, interagindo em todos os níveis de atenção em saúde (Brasil, 2002). Dentre as competências e habilidades de atuação profissional 
está a atuação em ações "de prevenção, promoção, proteção e reabilitação da saúde, tanto em nível individual quanto coletivo" (Brasil, 2002, p. 1), tendo como objetivo norteador o sistema de saúde do país (Ceccim e Carvalho, 2006).

Feuerwerker (2002) destaca o quanto as concepções econômicas e políticas do país influenciam as alterações curriculares. Diante deste cenário, as Diretrizes Curriculares se baseiam nos pilares da educação, descritos no relatório da Organização das Nações Unidas para a Educação, a Ciência e a Cultura (Unesco) em 1996, conhecido como Relatório Delors. Este relatório descreve que “a educação ao longo da vida" fundamenta-se em quatro pilares: "aprender a conhecer, aprender a fazer, aprender a conviver e aprender a ser" (Unesco, 2010, p. 31). Atualmente, a imagem-objetivo do fisioterapeuta é de um profissional que saiba conviver com outros profissionais, que conheça as necessidades de saúde dos locais onde atua, intervindo de modo preventivo e no processo de desenvolvimento do local, considerando a integralidade da atenção e a cultura de onde estão inseridos (Maranhão, 2003).

Ao resgatar-se a história da formação em Fisioterapia, reforça-se a concepção do quanto esta profissão ainda é considerada jovem, em fase de implementação e, principalmente, de fortalecimento profissional. Analisar a distribuição das escolas de Fisioterapia no Brasil provoca o pensamento sobre o futuro, não somente sobre a ótica do ensino, mas também em relação ao acesso aos profissionais fisioterapeutas, o perfil de egresso formado e, consequentemente, o modelo de atenção fornecido à população. Portanto, este artigo se propõe a analisar a distribuição de escolas de Fisioterapia nas diferentes regiões do Brasil, identificando as instituições com ensino nas modalidades presencial e a distância.

\section{Metodologia}

$\mathrm{O}$ estudo apresentado neste artigo ${ }^{4}$ classifica-se como quantitativo, exploratório, transversal, o qual utilizou fontes secundárias fornecidas no site e-MEC no primeiro semestre de 2015, nas quais constam todas as instituições de ensino superior cadastradas.

A busca identificou as escolas que oferecem ensino presencial e ensino a distância (EAD); e em atividade ou em processo de extinção (quando o curso não possui novos processos seletivos para ingresso) ou extintos. As informações foram coletadas no mês de maio de 2015 e relacionados o número de vagas e a distribuição de escolas, conforme estimativa populacional realizada pelo Instituto Brasileiro de Geografia e Estatística (IBGE).

Os dados foram tabulados em programa Microsoft Windows Excel 2010. A análise dos resultados está expressa em mapas elaborados no programa Datum Sirgas 2000 e em quadros. No intuito de atender aos critérios éticos, este estudo foi aprovado pelo Comitê de Ética em Pesquisa da Univates, conforme regulamentos da portaria ministerial n. 466, de 2012, do Ministério da Saúde, e aprovado sob o parecer n. 791.243, de 20 de setembro de 2014. 


\section{Resultados e discussão}

A análise do registro das instituições de ensino superior que constam no Ministério da Educação (MEC) aponta a desigualdade na oferta de cursos de Fisioterapia no Brasil. O crescimento vertiginoso do número de escolas e, consequentemente, do número de vagas resultaram também no fechamento de cursos que estão em processo de extinção ou já foram extintos.

O estado com maior número de cursos registrados é São Paulo, seguido de Minas Gerais e Paraná. Somados, os três estados concentram 45,6\% do total de registros das escolas de Fisioterapia no país (Tabela 1). O total daqueles cadastrados no MEC em 2015 foi de 797, ou seja, obteve-se um crescimento de $135 \%$ em pouco mais de dez anos naqueles de ensino superior, na área da Fisioterapia no Brasil. Andrade, Lemos e Dall'ago (2006) destacam que em 1991 o Brasil tinha 48 cursos de Fisioterapia, e 339 em 2004, um aumento de 706,25\%.

\section{Tabela 1}

\begin{tabular}{|c|c|c|c|c|c|}
\hline \multicolumn{6}{|c|}{ Distribuição de instituições de ensino de Fisioterapia por estado } \\
\hline Estado & N. Escolas & Em atividade & $\begin{array}{c}\text { Em extinção/ } \\
\text { Extinto }\end{array}$ & $\begin{array}{c}\text { Cursos } \\
\text { autorizados } \\
\text { EAD } \\
\end{array}$ & $\begin{array}{c}\text { Cursos } \\
\text { autorizados } \\
\text { presenciais }\end{array}$ \\
\hline Acre & 5 & 4 & 1 & 3 & 2 \\
\hline Alagoas & 16 & 14 & 2 & 3 & 13 \\
\hline Amazonas & 9 & 7 & 2 & 1 & 8 \\
\hline Amapá & 4 & 4 & 0 & 0 & 4 \\
\hline Bahia & 67 & 65 & 2 & 4 & 63 \\
\hline Ceará & 31 & 23 & 8 & 6 & 25 \\
\hline Distrito Federal & 20 & 14 & 6 & 7 & 13 \\
\hline Espírito Santo & 25 & 19 & 6 & 7 & 18 \\
\hline Goiás & 43 & 29 & 14 & 14 & 29 \\
\hline Maranhão & 11 & 8 & 3 & 2 & 9 \\
\hline Mato G. do Sul & 13 & 13 & 0 & 1 & 12 \\
\hline Mato Grosso & 13 & 13 & 0 & 2 & 11 \\
\hline Minas Gerais & 152 & 85 & 67 & 46 & 106 \\
\hline Pará & 18 & 13 & 5 & 3 & 15 \\
\hline Paraíba & 17 & 15 & 2 & 2 & 15 \\
\hline Paraná & 120 & 52 & 68 & 65 & 55 \\
\hline Pernambuco & 36 & 28 & 8 & 8 & 28 \\
\hline Piauí & 18 & 16 & 2 & 2 & 16 \\
\hline Rio de Janeiro & 92 & 51 & 41 & 34 & 58 \\
\hline Rio G. do Norte & 17 & 12 & 5 & 3 & 14 \\
\hline Rio G. do Sul & 85 & 46 & 39 & 38 & 47 \\
\hline Rondônia & 18 & 15 & 3 & 6 & 12 \\
\hline Roraima & 2 & 2 & 0 & 1 & 1 \\
\hline Santa Catarina & 30 & 15 & 15 & 13 & 17 \\
\hline São Paulo & 239 & 216 & 23 & 19 & 220 \\
\hline Sergipe & 11 & 9 & 2 & 2 & 9 \\
\hline Tocantins & 9 & 9 & 0 & 1 & 8 \\
\hline Totais & 1121 & 797 & 324 & 293 & 828 \\
\hline
\end{tabular}

Fonte: Brasil (2015).

Nota: EAD - Ensino a distância. 
Ao se analisarem os cursos em atividade, os estados de São Paulo e Minas Gerais permanecem com o maior número, seguidos pelo estado da Bahia. Somados, estes estados possuem $45,9 \%$ dos de escolas de fisioterapia em atividade no país. Na análise de concentração de cursos por estado, reitera-se o grande número de instituições de ensino em São Paulo, com 27,1\% do total de registrado (Figura 1).

Acerca da concentração dos cursos de ensino superior, Queiroz et al. (2013) destacam a aglomeração deles na região Sudeste (49\%), seguido pelo Nordeste, com $18 \%$ de todos da graduação. Este cenário revela o quanto o ensino superior se concentra na região de maior poder aquisitivo, emergindo as desigualdades regionais (Queiroz et al., 2013).

Ao se analisar o crescimento regional quanto ao número de cursos, identificou-se que ele foi mais expressivo na região Norte. Entretanto, salienta-se que não havia nenhum de Fisioterapia no Acre entre 1991 e 2004 (Andrade, Lemos e Dall'ago, 2006). No período de coleta de dados do presente estudo, o Acre possuía cinco registros: um em processo de extinção e quatro em atividade, com três registrados na modalidade a distância e dois presenciais.

Figura 1

Distribuição de escolas de Fisioterapia registradas (ativas ou extintas) no Brasil

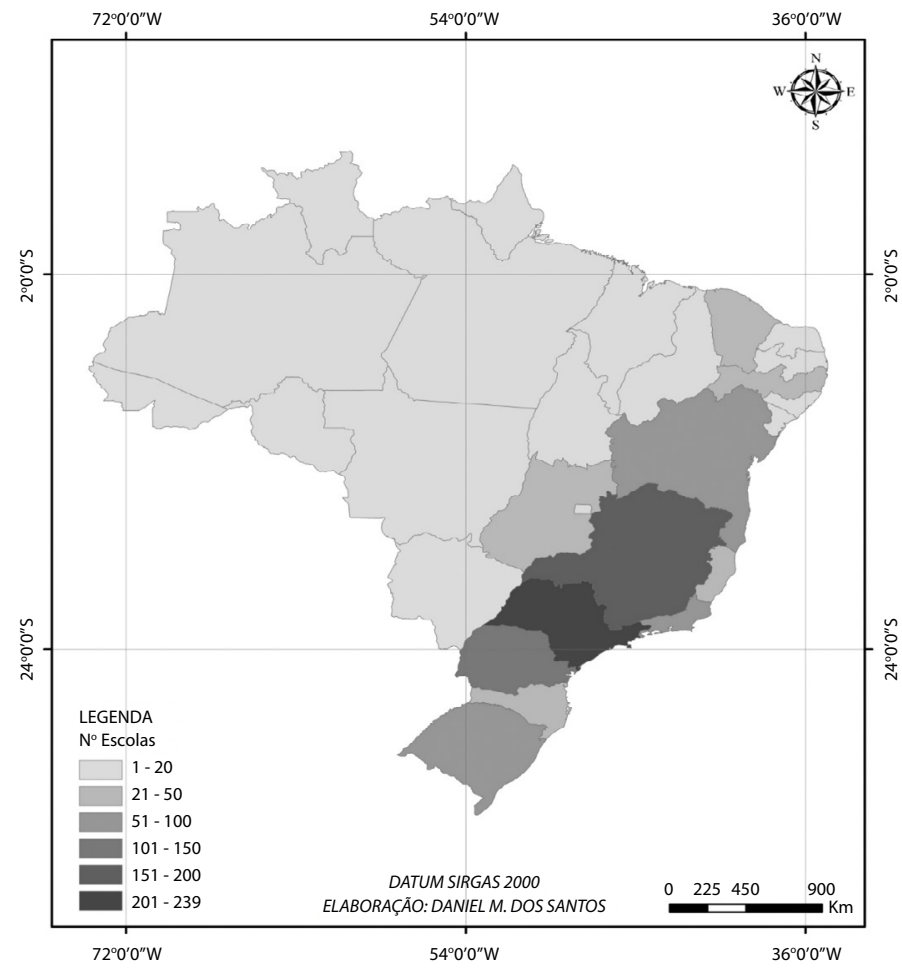

Fonte: Brasil (2015) 
Ao se analisar a distribuição de instituições de formação na área de Fisioterapia, identifica-se a discrepância entre as regiões. O Sudeste possui $46,5 \%$ do total de cursos em atividade, seguido pela região Nordeste com $23,8 \%$ e pela região Sul, com 14,2\% de instituições de ensino superior (Tabela 2).

\section{Tabela 2}

Distribuição de instituições de ensino de Fisioterapia por região e percentual de escolas em processo de extinção ou extintas e em atividade

\begin{tabular}{lccc}
\hline Região & Em atividade & Em extinção/Extinto & $\begin{array}{c}\text { Percentual de escolas } \\
\text { em processo de } \\
\text { extinção ou extintas (\%) }\end{array}$ \\
\hline Nordeste & 190 & 34 & 15,2 \\
Norte & 54 & 11 & 16,9 \\
Centro-Oeste & 69 & 20 & 22,5 \\
Sudeste & 371 & 137 & 27,0 \\
Sul & 113 & 122 & 51,9 \\
\hline
\end{tabular}

Fonte: IBGE (2015).

Considerando o número de vagas a cada 10 mil habitantes por região, a concentração na região Sudeste fica ainda mais evidente. São 4,54 vagas a cada 10 mil habitantes, seguido pela região Centro-Oeste com 3 vagas e região Sul, com 2,15 vagas a cada 10 mil habitantes. Apesar do crescimento no número de cursos na região Nordeste, a disponibilidade de vagas para estudantes de Fisioterapia é de somente 0,98 vagas a cada 10 mil habitantes, e no Norte, o total de vagas é de 1,25 (Tabela 3).

A análise de proporção de vagas por habitantes revela uma realidade brasileira. Taneguti (2012) destaca que, mesmo com as políticas de incentivo para criação de vagas no ensino superior, o acesso é privilégio de uma minoria e, segundo a autora, menos de $5 \%$ da população teve acesso às IES. Apesar do aumento significativo no número de escolas, a taxa de escolarização de pessoas entre 18 e 24 anos é considerada um problema. Em 2010, a média de estudantes no ensino superior foi de 13,6\% no Brasil. Na região Sul, 20\% dos jovens estão no ensino superior, enquanto nas regiões Norte e Nordeste este índice é de 9,9\%. Em relação ao número de vagas, Haddad et al. (2010) analisaram a distribuição de vagas de 14 cursos da área da saúde, cujos resultados corroboraram os dados encontrados neste trabalho, onde $57 \%$ dos concluintes dos cursos da área da saúde estavam na região Sudeste do Brasil.

O número de vagas ofertadas para o ensino superior ainda é menor em relação à procura dos estudantes. Em 2011 foram ofertadas 68.939 vagas para os cursos de Fisioterapia, e o número de inscritos foi de 137.102 pessoas. Todavia, há discrepância entre o número de vagas entre instituições públicas e privadas. Das 68 mil vagas ofertadas, somente 4.043 eram de instituições públicas, o que representou $5,86 \%$ de todas as vagas no Brasil. Em contrapartida, o número 
de pessoas que se inscreveram para os processos seletivos nas instituições públicas foi de 34,77\% (47.683) do total de inscritos (Taneguti, 2012).

Tabela 3

\begin{tabular}{lccc}
\hline \multicolumn{4}{l}{ Distribuição de vagas nos cursos de Fisioterapia por região brasileira } \\
\hline Região & População & Vagas & Vagas/10mil \\
\hline Norte & 17.231 .027 & 2.160 & 1.25 \\
Nordeste & 56.186 .190 & 5.505 & 0.98 \\
Sudeste & 85.115 .623 & 38.655 & 4.54 \\
Sul & 29.016 .114 & 6.251 & 2.15 \\
Centro-Oeste & 15.219 .608 & 4.562 & 3.00 \\
\hline
\end{tabular}

Fonte: INEP (2006); IBGE (2015).

Acompanhando o crescimento nacional de vagas em cursos da área da saúde, reflexo de políticas entre 1991 e 2008, o número de cursos de Fisioterapia no Brasil cresceu $658 \%$ nesse mesmo período (Haddad et al., 2010). Consequentemente, o número de vagas nos cursos da área da saúde aumentou consideravelmente, reflexo de políticas desenvolvidas ainda nas décadas de 1960 e 1970. Todavia, o crescimento desenfreado no número de escolas acentuou o desequilíbrio regional em relação ao surgimento das escolas da área da saúde. É necessário atenuar estes desequilíbrios regionais e investir na criação de vagas nas áreas com menor concentração. As vagas devem ser ofertadas observando-se as necessidades epidemiológicas da população, articulando-se a formação profissional ao sistema de saúde (Haddad et al., 2010).

Neste cenário, o número de vagas tornou-se insustentável, impactando no fechamento das escolas e no surgimento de outra modalidade de ensino, o semipresencial ou a distância. O EAD ainda se configura como um desafio para o ensino de uma profissão, principalmente no que se refere à área da saúde. Em contraponto, também é considerada como uma potencialidade, na medida em que facilita o acesso de um maior número de pessoas para o ensino superior. O EAD está previsto no decreto n. 5622/2005 e estabelece algumas regras para cursos que possuem especificidades, como a Fisioterapia. Neste sentido, não há EAD propriamente dito, mas encontros semipresenciais, já que o desenvolvimento de estágios e de práticas exige uma infraestrutura adequada para este tipo de ensino (Brasil, 2005).

Na coleta dos dados, no que tange a essa modalidade, identificou-se que os cursos de graduação em Fisioterapia estão concentrados em apenas cinco instituições. Quando analisados somente os cursos registrados na modalidade EAD, foram encontrados 293 registros. Destes, $26 \%$ estão em fase de extinção ou extintos e 40 em atividade.

Os cursos em atividade de EAD estão concentrados em 15 estados: no Acre e em Mato Grosso, disponíveis dois cursos na modalidade semipresencial; 
em Alagoas, Espírito Santo, Goiás, Minas Gerais, Mato Grosso do Sul, Paraná, Roraima, Rio Grande do Sul e Tocantins e no Distrito Federal, há somente um curso de Fisioterapia a distância em cada estado. Na Bahia, são três, já em Roraima há cinco cursos ofertados neste modelo, e o estado com maior concentração na modalidade a distância é São Paulo, com 18 cursos de graduação.

$\mathrm{O}$ crescimento desse tipo de curso é uma realidade. O número de estudantes que cursavam graduação a distância em 2002 era de 40.714, passando para 930.179 em 2010 (Queiroz et al., 2013), todos ofertados em instituições privadas de ensino superior.

$\mathrm{O}$ EAD apresenta-se como uma alternativa de acesso da população à educação superior, com vistas à diminuição das desigualdades sociais e à disseminação das informações. Entretanto, para seu desenvolvimento, são necessários alguns cuidados, pois antes de considerá-lo como uma 'tábua de salvação' para o acesso à informação, tal como ocorre no ensino presencial, é o aluno quem deve ser o protagonista de sua aprendizagem. Independentemente da modalidade de ensino, são os estudantes que devem elaborar suas próprias perguntas, desenvolver sua postura crítica e reflexiva perante as situações (Becker e Marques, 2002). Porém, a imagem, por si só, não fará o EAD. A redução do ensino como um reprodutor de imagens, algo a ser transmitido de uma pessoa para outra, representa um nó a ser superado. A aprendizagem ocorre na construção de perguntas, nas discussões e estranhamentos, essa é a potência do ensino, seja ela presencial ou a distância (Becker e Marques, 2002).

Sobre o EAD, Santos (2008) destaca seu impacto na proliferação das informações e possibilidades de ensino e aprendizagem. A superação da universidade como um espaço territorial e das formas tradicionais de ensino é um resultado concreto da atualidade. Ainda é necessário perceber como esta transformação impactará na pesquisa, na extensão universitária e na formação, uma vez que não há espaços concretos, mas virtuais. Santos teria afirmado naquele trabalho (2008) não considerar a modalidade à distância melhor ou pior do que o ensino presencial; porém, este modo de ensinar deve ser ponderado e analisado conforme a profissão desejada.

No tocante ao registro das escolas, dos 1.121 identificados, 28,9\% estão em processo de extinção ou já foram extintos (Figura 2), sobressaindo os estados do Paraná e Minas Gerais com 56,7\% e 44,1\%, respectivamente.

No que se refere ao percentual de cursos de Fisioterapia em processo de extinção ou já extintos, destaca-se a região Sul, onde 51,9\% deles estão encerrando ou encerraram suas atividades, pelas regiões Sudeste e Centro-Oeste, com $26,9 \%$ e $22,47 \%$ dos cursos fechados, respectivamente. Apesar das regiões Norte e Nordeste apresentarem menor concentração de cursos fechados, estão em processo de encerramento de suas atividades $16,9 \%$ e $15,2 \%$ dos cursos das regiões, respectivamente. 
Figura 2

Concentração de cursos em processo de extinção ou extintos no Brasil

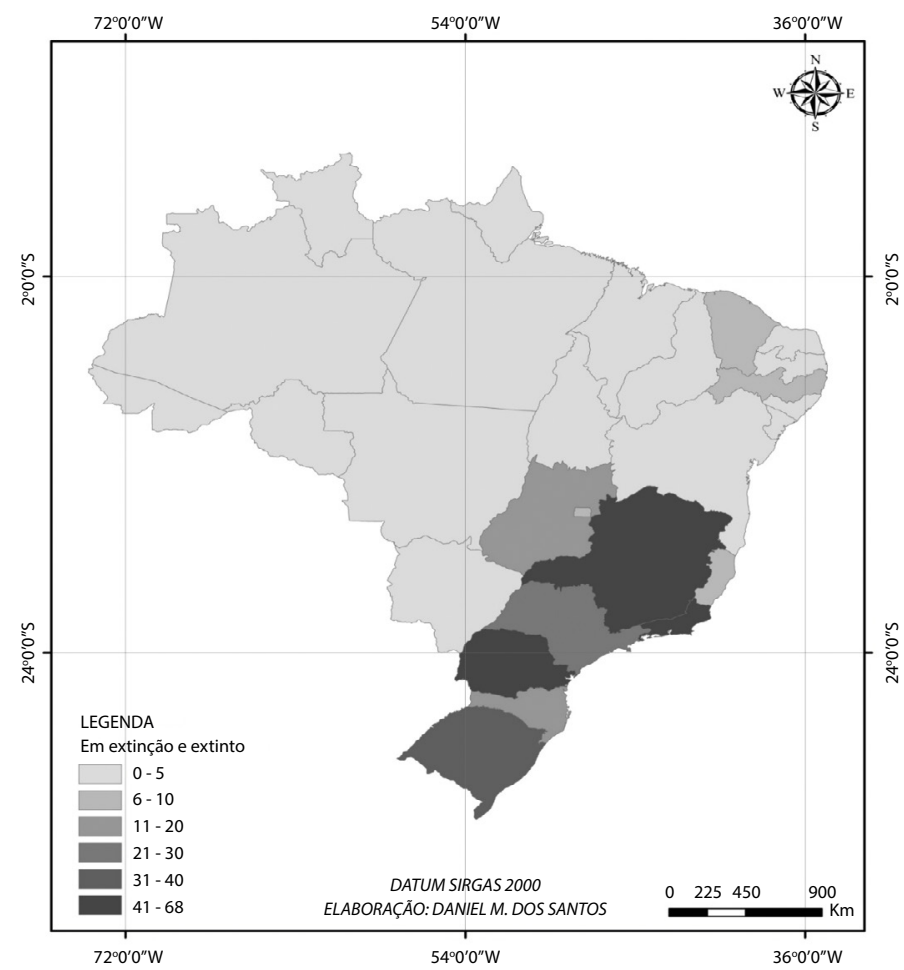

Fonte: Brasil (2015).

O aumento no número dos cursos em processo de extinção ou extintos não se deve ao excesso de profissionais da área, mas sim ao reflexo do crescimento desenfreado dos cursos de Fisioterapia no Brasil. As regiões Sul e Sudeste possuem o maior número de cursos que estão em processo de extinção ou extintos (Tabela 3). No período de 1996, houve um crescimento intenso no credenciamento de cursos nas duas regiões (Haddad et al., 2010).

\section{Conclusão}

Este artigo debruçou-se sobre a distribuição de escolas de Fisioterapia nas diferentes regiões do Brasil, identificando as instituições que oferecem cursos nas modalidades presencial e a distância. A concentração de escolas no estado de São Paulo é reflexo da ausência de planejamento da abertura de novos cursos. Enquanto isso, os estados da região Norte ainda necessitam de investimento na abertura de novos cursos de formação, apesar de apresentarem o maior crescimento regional. 
O grande número de extinção de cursos parece revelar uma 'crise' na área da Fisioterapia, entretanto identifica-se o quanto a atuação profissional ainda está concentrada na atuação terciária e em grandes centros populacionais. A formação em Fisioterapia só atinge o interior do Brasil quando analisados os cursos EAD.

Planejar e organizar a formação no Brasil sempre foi um nó crítico. O crescimento vertiginoso de escolas de Fisioterapia, somado à falta de critérios para a abertura de novos cursos no país, reitera as desigualdades na distribuição de profissionais.

A falta de discussão em torno do ensino a distância também corrobora a falta de decisão e de autonomia dos cursos. Se, por um lado, a tecnologia, cada vez mais presente no cotidiano das pessoas, aproxima e facilita o acesso à informação, por outro, há a falta de critérios e de discussão das especificidades para a formação, especialmente em relação aos cursos da área da saúde. Enquanto na área da Fisioterapia instituições como a Associação de Ensino em Fisioterapia condenam veementemente a criação de cursos na modalidade a distância, o Ministério da Educação autoriza as instituições de ensino a ofertarem cursos nesta modalidade.

Enquanto o EAD ganha status de problema para a formação de fisioterapeutas, não se pode negar que o emprego da tecnologia deva estar cada vez mais presente no ensino. Evocamos a discussão, a análise dos fatos e a construção de outros caminhos, nas quais quem ganha é a formação dos profissionais e consequentemente a saúde da população.

\section{Colaboradores}

Lydia Chrismann Espíndola Koetz participou da coleta e análise dos dados, descrição e análise dos resultados e elaboração do texto final. Eduardo Périco orientou a coleta de dados, a análise dos resultados e revisão do texto final. Magali Quevedo Grave orientou a análise dos resultados e revisão do texto final. 
Resumen El crecimiento vertiginoso en el número de escuelas de Fisioterapia en Brasil carece de análisis. Entre 1995 y 2008 hubo un aumento del 476\% en el número de cursos localizados en grandes ciudades y en instituciones de enseñanza privadas. Con el objetivo de analizar la distribución de escuelas de Fisioterapia en las diferentes regiones brasileñas, identificando las instituciones con enseñanza presencial y a distancia, se realizó un estudio cuantitativo, exploratorio, transversal, a partir de datos secundarios del sitio e-MEC en 2015. Se listaron el número de vacantes y la distribución de las escuelas, según población estimada. Los cursos de Fisioterapia están concentrados en grandes ciudades, dificultando el acceso de la población del interior. La mayor concentración tiene lugar en los estados de San Pablo (239 cursos), Minas Gerais (152 cursos) y Paraná (120 cursos), donde también se encuentra el mayor número de profesionales. En el análisis de la distribución de cursos, se identificó la necesidad de invertir en la apertura de cursos en la región Norte. El crecimiento desenfrenado de escuelas de Fisioterapia, sumado a la falta de criterios para la apertura de nuevos cursos en el país, reitera las desigualdades en la distribución de profesionales.

Palabras clave fisioterapia; educación a distancia; enseñanza.

\section{Notas}

${ }^{1}$ Centro Universitário Univates, Lajeado, Rio Grande do Sul, Brasil.

$<$ lkoetz@gmail.com>

Correspondência: Rua Avelino Talini, 171, CEP 95900-000, Lajeado, Rio Grande do Sul, Brasil.

${ }^{2}$ Centro Universitário Univates, Lajeado, Rio Grande do Sul, Brasil.

<perico@univates.br>

${ }^{3}$ Centro Universitário Univates, Lajeado, Rio Grande do Sul, Brasil.

<mgrave@univates.br>

${ }^{4}$ Este artigo é resultado da tese intitulada Do ambiente da doença à inserção na saúde: perspectiva da Fisioterapia no cenário brasileiro, de autoria de Lydia Chrismann Espíndola Koetz, apresentada no ano de 2015, ao Programa de Pós-Graduação stricto sensu em Ambiente e Desenvolvimento do Centro Universitário Univates, Lajeado, Rio Grande do Sul. 


\section{Referências}

ANDRADE, Armèle D.; LEMOS, Jadir C.; DALL'AGO, Pedro. Fisioterapia. In: HADDAD, Ana E. et al. (org.). A trajetória dos cursos de graduação na área da saúde: 1991-2004. Brasília: Instituto Nacional de Estudos e Pesquisas Educacionais Anísio Teixeira, v. 15, 2006. p. 203-241.

BECKER, Fernando; MARQUES, Tania B. I. Ensino ou aprendizagem a distância. Educação em Revista, Curitiba, n. 19, p. 85-98, jun. 2002. Disponível em <http://www. scielo.br/scielo.php?script $=$ sci_arttext\&pi$\mathrm{d}=$ S0104=40602002000100007-\&lng=pt\&nrmiso >. Acesso em: 18 jul. 2015.

BISPO JÚNIOR, José P. Formação em fisioterapia no Brasil: reflexões sobre a expansão do ensino e os modelos de formação. História, Ciências, Saúde: Manguinhos, Rio de Janeiro, v. 16, n. 3, set. 2009. Disponível em: <http://www. scielo.br/scielo.php?script $=$ sci_arttext \&pi$\mathrm{d}=$ S0104=59702009000300005-\&lng=en\&nrmiso >. Acesso em: 20 maio 2014.

BISPO JÚNIOR, José P. Trajetória da fisioterapia no Brasil. In: BISPO JÚNIOR, José P. (org.). Fisioterapia \& Saúde Coletiva: reflexões, fundamentos e desafios. São Paulo: Hucitec, 2013. p. 17-49.

BRASIL. Conselho Nacional de Educação, Câmara de Educação Superior. Resolução n. CNE/CES n. 4, de 19 de fevereiro de 2002. Institui diretrizes curriculares nacionais do curso de graduação em Fisioterapia. Brasília, DF: MEC, 2002.

BRASIL. Decreto-lei n. 5.622, de 19 de dezembro de 2005. Regulamenta o art. 80 da lei n. 9.394, de 20 de dezembro de 1996, que estabelece as diretrizes e bases da educação nacional. Ministério da Educação e Cultura. Brasília, DF, 2005. Disponível em: <http://www.planalto.gov.br/ ccivil_03/_Ato2004-2006/2005/Decreto/D5622. htm>. Acesso em: 23 jul. 2015.

BRASIL. E-MEC. Instituições de Educação Superior e Cursos Cadastrados. 2015. Dispo- nível em: <http://emec.mec.gov.br/> . Acesso em: 23 jul. 2015

CECCIM, Ricardo B; CARVALHO, Yara M. Ensino da saúde como projeto da integralidade: a educação dos profissionais de saúde no SUS. In: PINHEIRO, Roseni; CECCIM, Ricardo B.; MATTOS, Ruben A. (orgs.). Ensinar saúde: a integralidade e o SUS nos cursos de graduação da área da saúde. Rio de Janeiro: Lappis, 2006. p. 69-92.

FEUERWERKER, Laura. Além do discurso de mudança na educação médica: processos e resultados. Rio de Janeiro; São Paulo: Associação Brasileira de Educação Médica; Hucitec, 2002.

HADDAD, Ana E. (org.). A trajetória dos cursos de graduação na saúde - 1991 a 2004. Brasília: Instituto Nacional de Estudos e Pesquisas Educacionais Anísio Teixeira, 2006

HADDAD, Ana E. et al. Formação de profissionais de saúde no Brasil: uma análise no período de 1991 a 2008. Revista de Saúde Pública, São Paulo, v. 44, n. 3, p. 383-393, jun. 2010. Disponível em: <http://www. scielo.br/scielo.php?script=sci_arttext\&pi$\mathrm{d}=$ S0034=89102010000300001-\&lng=en\&nrmiso >. Acesso em: 14 jul. 2015.

INSTITUTO NACIONAL DE ESTUDOS E PESQUISAS EDUCACIONAIS ANÍSIO TEIXEIRA (INEP). 2006. Disponível em: < http://www. publicacoes.inep.gov.br/arquivos/\%7BDA44B69A-B6B3-427F-8095-CF35F478533A\%7D_Fisioterapia.pdf>. Acesso em: 20 maio 2014.

INSTITUTO NACIONAL DE GEOGRAFIA E ESTATÍSTICA (IBGE). Estimativas populacionais para os municípios e para as Unidades da Federação brasileiros. Disponível em: $<$ http://www.ibge.gov.br/home/estatistica/ populacao/estimativa2016/estimativa_dou. shtm>. Acesso em: 23 jul. 2015.

MARANHÃO, Efrem A. [Prefácio] A construção coletiva das diretrizes curriculares nacionais dos cursos de graduação em saúde: uma con- 
tribuição para o Sistema Único de Saúde. In: ALMEIDA, Marcio. Diretrizes Curriculares Nacionais para os Cursos Universitários da Área da Saúde. Londrina: Rede Unida, 2003. p.12.

OLIVEIRA, V.R.C. A História dos currículos de Fisioterapia: A construção de uma identidade profissional. 2002. Dissertação (Mestrado em Educação) - Universidade Católica de Goiás, Goiânia, 2002.

QUEIROZ, Fernanda C. B. P. et al. Transformações no ensino superior brasileiro: análise das Instituições Privadas de Ensino Superior no compasso com as políticas de Estado. Ensaio: Avaliação e Políticas Públicas em Educação, Rio de Janeiro, v. 21, n. 79, p. 349-370, abr.-jun. 2013. Disponível em: <http://www.scielo.br/scielo. php?pid=S0104=40362013000200009-\&scriptsci_arttext>. Acesso em: 18 jul. 2015.

SANTOS, Boaventura S. Universidade no século XXI: para uma reforma democrática e emancipatória da universidade. In: SANTOS,
Boaventura S.; ALMEIDA-FILHO, Naomar. $A$ universidade do século XXI: para uma universidade nova. Coimbra: Almedina, 2008.

TANEGUTI, Luiza Y. Desenvolvimento, aprimoramento e consolidação de umaeducação nacional de qualidade. Projeto CNE/Unesco. Relatório técnico.Brasília: Ministério da Educação, 2012. Disponível em: <http://portal.mec.gov. br/index.php?option $=$ com_docman \&view $=$ download\&alias = 13948-produto-2-oferta-demanda-educ-superior-pdf-pdf\&category_slu$\mathrm{g}=$ setembro-2013-pdf\&Itemid=30192 > . Acesso em: 18 jul. 2015

UNESCO. Um tesouro a descobrir: relatório para a Unesco da Comissão Internacional sobre a Educação para o século XXI. Brasília: Unesco, 2010. 41 p.

Recebido em 17/03/2016

Aprovado em 13/07/2016 
